\title{
Towards Energy Consumption Measurement in a Cloud Computing Wireless Testbed
}

\author{
Vitor Bernardo, Marilia Curado \\ Center for Informatics and Systems \\ University of Coimbra \\ Polo II, Pinhal de Marrocos, \\ 3030-290 Coimbra, Portugal \\ E-mail: \{vmbern, marilia\}@dei.uc.pt
}

\author{
Thomas Staub, Torsten Braun \\ Institute for Computer Science \\ and Applied Mathematics \\ University of Bern \\ Neubrückstrasse 10, CH-3012 Bern, Switzerland \\ E-mail: $\{$ staub , braun $\} @$ iam.unibe.ch
}

\begin{abstract}
The evolution of the Next Generation Networks, especially the wireless broadband access technologies such as Long Term Evolution (LTE) and Worldwide Interoperability for Microwave Access (WiMAX), have increased the number of "allIP" networks across the world. The enhanced capabilities of these access networks has spearheaded the cloud computing paradigm, where the end-users aim at having the services accessible anytime and anywhere. The services availability is also related with the end-user device, where one of the major constraints is the battery lifetime. Therefore, it is necessary to assess and minimize the energy consumed by the end-user devices, given its significance for the user perceived quality of the cloud computing services. In this paper, an empirical methodology to measure network interfaces energy consumption is proposed. By employing this methodology, an experimental evaluation of energy consumption in three different cloud computing access scenarios (including WiMAX) were performed. The empirical results obtained show the impact of accurate network interface states management and application network level design in the energy consumption. Additionally, the achieved outcomes can be used in further software-based models to optimized energy consumption, and increase the Quality of Experience (QoE) perceived by the endusers.
\end{abstract}

Index Terms-Cloud Computing, Energy, Measurement, Testbed, Wireless, 4G networks.

\section{INTRODUCTION}

Next Generation Network (NGN) [1] is the common term to describe a packet-based broadband network, designed to support all services and information exchange transparently under all the available networks. Since the communication is based on packet encapsulation, and since IP is widely deployed, the term "all-IP" network is commonly associated with the development of a NGN.

This emerging era of access networks that is being spearheaded by the $4 \mathrm{G}$ systems has as main objective the creation of high speed wireless technologies based on an all-IP approach, which will grant better performance and optimize the communication with IP-based core systems such as the Internet. The optimization of the $4 \mathrm{G}$ systems such as Long Term Evolution (LTE), Worldwide Interoperability for Microwave Access (WiMAX), or Evolved High-Speed Packet Access (HSPA+) together with the widely used wireless technology IEEE 802.11, provides connectivity to global wireless communication systems that have different capabilities and costs.
Together with the fast evolution of access technologies, the cloud computing paradigm has emerged, and several discussions in the community regarding the cloud definition have occurred [2]. There is no clear agreement in the community about the cloud computing service types, as they are related with the users or brokers that are accessing them. Depending on the type of usage / capacity provided, the cloud scenarios are commonly classified into Infrastructure as a Service (IaaS), Platform as a Service (PaaS) and Software as a Service (SaaS). Concerning the SaaS, many multimedia based applications have arose during the last years, with more and more people using them. Due to the characteristics of these types of applications, user demands from the access technology will also increase. Therefore, it is important for both operator and enduser to measure the applications quality in order to assess the capabilities of a certain network to provide the needed quality. However, the quality assessment is historically associated with the evaluation of some parameters at the network layer. The common metrics associated with the Quality of Service (QoS), namely the available bandwidth, delay or packet loss rate, cannot represent the real quality perceived by the endusers at the application level. To overcome this limitation, the novel concept of the Quality of Experience (QoE) has been developed. By employing QoE-aware techniques, it is possible to understand the real perception of the end-user, providing complementary information to the more common QoS parameters.

The successful deployment of a NGN will also encompass a considerable number of wireless devices moving with different speeds, patterns and communicating through various radio interfaces. In a NGN with various technologies involved, the number of different wireless access technologies is also growing and support to seamless vertical handovers within heterogeneous networks is required [3]. Regarding the handover, the first important initial step is the network selection. Currently, many network approaches are solely based on the Received Signal Strength (RSS) [4], which is very limited. Other approaches, such as multi-criteria cost-based function, based on fuzzy logic [5] or neural networks [6] are also being employed as strategies in the network selection process.

Another parameter that has to considered is the device energy 
consumption. In fact, the energy consumption becomes an important end-user experience parameter, because end-users aim to maximize the device battery life. This optimization can include the application performance optimization, but should also take into account the energy consumption in both wireless interfaces and network protocols. The NGN heterogeneity together with the fast deployment of all the applications to the cloud and the usage of many applications as a service, takes the handover optimization problem to the application level, where the network interface energy consumption can be one of the key mobility decision factors.

This work proposes an empirical methodology for assessing the energy consumption of a network interface using high precision measurement hardware. The gathered data shows important relationships between applications and energy consumption. It can be used to develop software-based energy models that be can be employed to optimize energy consumption and QoE in mobile cloud computing scenarios with heterogeneous technologies available.

The remaining sections of this paper are organized as follows. Section II describes the proposed methodology for energy consumption assessment and the cloud computing testbed. The analysis of experimental evaluation results is presented in Section III, followed by the related work discussion in Section IV. Finally, Section V presents the main conclusions and contributions drawn from the empirical assessment performed.

\section{Methodology}

This section describes the employed methodology for the measurement of energy consumption in a cloud computing device and the cloud computing testbeds used.

\section{A. Objectives}

This measurement methodology aims to fulfill a set of requirements for the assessment of the energy consumption in a cloud computing scenario, namely:

- Testbed assessment: since energy consumption in simulators is only based on models, it is important to perform testbed assessments in order to accurately measure the energy impact in real life systems;

- High-precision measurements: to guarantee a good accuracy of testbed energy measurements it is vital to use a hardware capable to support multiple samples per second, since energy in small devices (i.e. network interfaces) tends to have slight variations along the time;

- Independent network interface evaluation: to better understand the impact of the network interface in the energy consumption, it is essential to limit the measurements to the network interface lower layers, namely by assessing the energy utilization in MAC and PHY layers. These layers are the only not directly related with the device and operating system;

- States: to study the different states used in the each network technology, as good manipulation of these states can also contribute to energy savings.

\section{B. Power measurement setup}

The energy measurement testbed was designed to meet all requirements mentioned above, and making only mandatory changes to the real system hardware. The first option was to use an external USB network interface, since it is possible to measure accurately the energy consumed solely by the interface, as desired. One of the main issues already reported in previous energy measurement works is the need to provide a stable and continuous voltage to the system [7] [8]. The impact on the voltage drawn of connecting the USB network interface directly to an end-user device was noticeable in the preliminary tests. To overcome this limitation, the USB network interface was connected to an external AC powered USB hub, able to give stable power to the system. The analysis regarding the voltage drawn when employing the external USB hub has shown voltage drops are always lower than $1 \%$ of the total employed voltage, which is negligible in the overall system analysis.

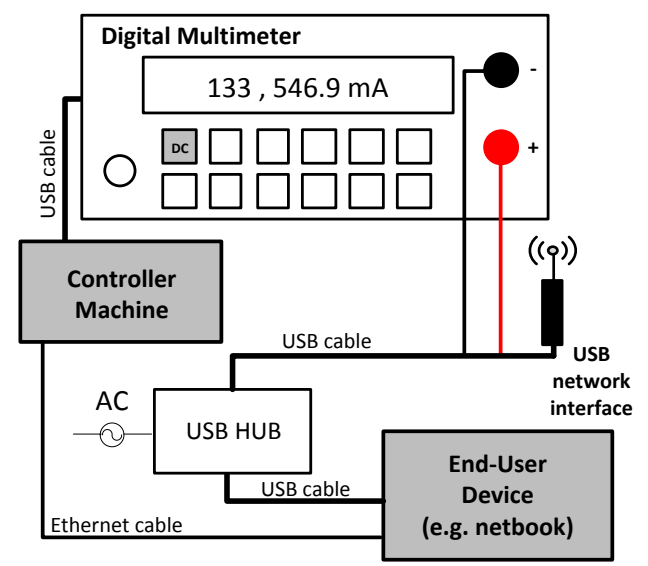

Fig. 1. Energy measurement setup

Figure 1 depicts the energy measurement testbed setup. Besides the end-user device (referred also as mobile node in the rest of this paper), the measurement configuration includes a "controller machine" and a high-precision digital multimeter. The digital multimeter is a Rigol DM3061 with a maximum sampling rate of $50 \mathrm{~K}$ samples/second and a test resolution of $61 / 2$ digits. The multimeter is capable of receiving Standard Commands for Programmable Instruments (SCPI) commands (defined by IEEE 488.2 [9]) and implements the Universal Serial Bus Test and Measurement Class Specification (USBTMC) standard interface.

By using SCPI commands and USBTMC the "controller machine" is able to control and manage the digital multimeter, which enables accurate and repeatable tests. The "controller machine" is also connected to the end-user device. This entity enables a fast and reliable point to control the experiments to be performed and to collect all the results from the digital multimeter.

Since the voltage is stable, all the measurements concerning energy were done by collecting the current values only. The 


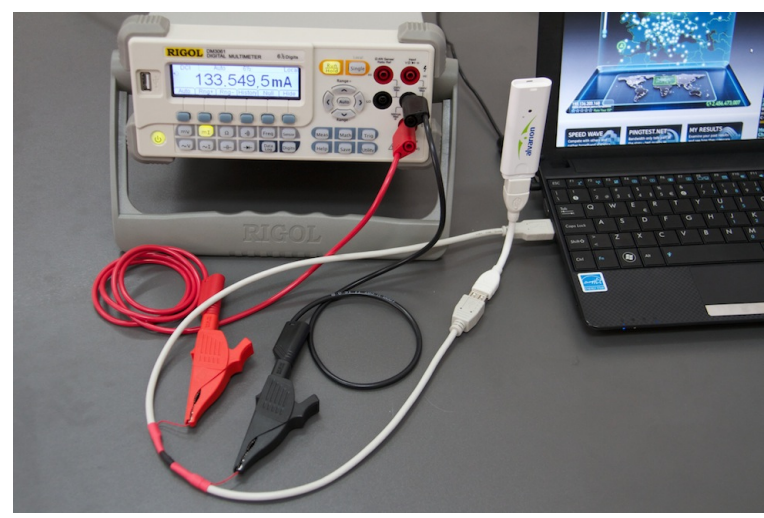

Fig. 2. Energy measurement testbed

USB cable was intercepted in the common-collector voltage (VCC) cable (i.e., +5 VDC), as illustrated in Figure 2.

\section{Cloud computing testbed}

This subsection presents the University of Coimbra IEEE 802.16e (Mobile WiMAX) and IEEE 802.11 (WiFi) testbeds. The IEEE 802.16e (Mobile WiMAX) testbed contains two Base Stations (BS) located in two distinct city areas and a set of Customer Premises Equipment (CPE) of different types, namely USB sticks, and indoor / outdoor units. However, as

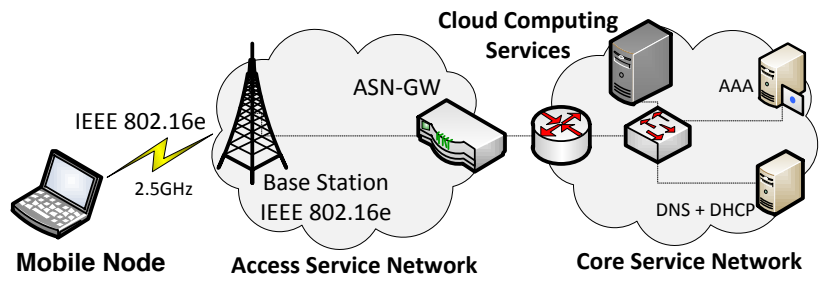

Fig. 3. IEEE 802.16e (Mobile WiMAX) testbed

depicted in Figure 3, only one base station was used in this work. The USB CPE, Alvarion USB BreezMAX 250, was used. The Mobile WiMAX BS is an Alvarion BreezeMAX Macro Outdoor Network Access Unit. The most relevant configuration parameters are the following:

- Central Frequency: 2610.00 MHz;

- Total uplink duration: 6 slots;

- Modulation: 64-QAM 5/6 (best possible);

- Antennas: two $65^{\circ}$ sector dual polarization antennas with maximum TX power of $38 \mathrm{dBm}$;

- Resource Reservation: Best Effort channel of 15Mbps.

The IEEE 802.16e testbed is fully compliant with the WiMAX Forum Network Reference Model [10], where all the WiMAX standard entities and relationships between them are defined. The IEEE 802.11 testbed is composed by a high performance IEEE 802.11n router, the Cisco Linksys E4200, and USB dualband $(2.4 \mathrm{GHz}$ and $5 \mathrm{GHz})$ network interface, the Cisco Linksys AE1000. The Cisco Linksys E4200 is a dual-band $(2.4 \mathrm{GHz}$ and $5 \mathrm{GHz}$ ) IEEE $802.11 \mathrm{n}$ router with Gigabit Ethernet ports, including also the support of Multiple-Input and MultipleOutput (MIMO) 3x3 and 6 internal antennas.
The IEEE 802.11 testbed is illustrated in Figure 4. An Asus EEE 1001PX-H netbook (CPU: Intel Atom N450 1.66 GHz ; RAM: $2 \mathrm{~Gb}$ ) was used as "Mobile Node" equipment. In all the experiments the netbook was running Ubuntu Linux kernel version 2.6.32-21-generic. The "Cloud Computing Services" machine located in the core network is a HP ProLiant DL320 G5p server (CPU: Intel Xeon X3210, 2.16GHz ; RAM: 4Gb) running Debian Linux kernel version 2.6.32-5-amd64.

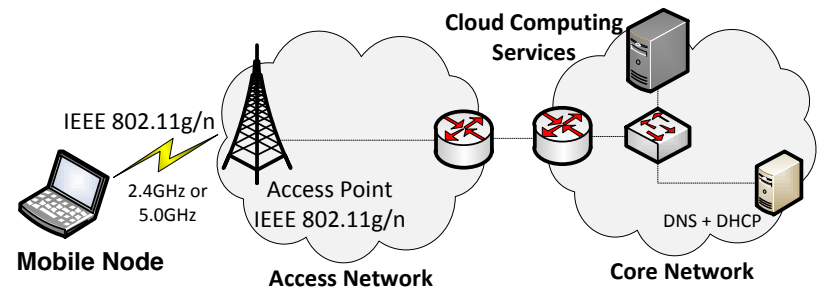

Fig. 4. IEEE 802.11 testbed

All energy measurements performed used the setup already explained in the previous section. The traffic referred as "receiving" is generated by the "Cloud Computing Services" machine in the core network and received by the "Mobile Node" in each scenario. The "transmitting" term is used to express the traffic with source on "Mobile Node" and with "Cloud Computing Services" as destination.

The "Cloud Computing Services" machine aims to represents all the services available for an end-user in a cloud computing scenario. This study is not focused on the services, but on accessing them in an energy efficient way.

\section{EXPERIMENTAL EVALUATION}

This section describes the experimental evaluation performed concerning energy consumption assessment in mobile cloud computing scenarios. The tests were performed in three different scenarios, as depicted in Table I.

TABLE I

EXPERIMENTAL EVALUATION SCENARIOS

\begin{tabular}{ll} 
Name & Description \\
\hline \hline WiMAX & $\begin{array}{l}\text { Tests performed using in the } \\
\text { IEEE } 802.16 \mathrm{e} \text { (Mobile WiMAX) testbed }\end{array}$ \\
\hline WiFi 2.4GHz & $\begin{array}{l}\text { Tests performed in the } \\
\text { IEEE } 802.11 \text { testbed at } 2.4 \mathrm{GHz}\end{array}$ \\
\hline WiFi 5.0GHz & $\begin{array}{l}\text { Tests done in the IEEE } 802.11 \text { testbed } \\
\text { using the 5GHz frequency }\end{array}$ \\
\hline
\end{tabular}

All results presented in the following sections are measured according to the defined energy measurement methodology, and include 15 runs for each test setup with a confidence interval of $95 \%$. The energy consumption was calculated by measuring the power consumption using a rate of 833 samples. These experiments did not use the $50 \mathrm{~K}$ samples rate, since the digital multimeter is only able to measure 43 seconds when using this rate.

Each test performed has a total duration of 120 seconds, 
whereas the first and the last 15 seconds of the experiment were not considered, in order to avoid the impact of the energy consumed by the User Datagram Protocol (UDP) socket establishment and release procedures. As a result, all the energy results presented only consider the energy consumed during them 90 seconds.

\section{A. Objectives}

The main objective of this experimental evaluation is to understand the relationship between the application design and the energy consumed by the network interface. Additionally, the energy demands of different network access technologies to be used in the cloud computing scenarios, namely the WiMAX and the WiFi (using two distinct frequency, namely $2.4 \mathrm{GHz}$ and $5.0 \mathrm{GHz}$ ) are also studied.

\section{B. Assessment of device states impact}

This subsection shows the impact of the various network states in the energy consumption. The different access technologies can have two different states:

- Disconnected: network interface is disconnected from the network (i.e. the radio was switched-off);

- Connected: network interface is associated with the network.

Figure 5 shows the energy consumption of the studied wireless access technologies in the previously defined states during 90 seconds. When connected to the network, the WiFi $5.0 \mathrm{GHz}$ has the highest energy requirements. Both WiFi $2.4 \mathrm{GHz}$ and WiMAX have similar performance in the connected state, but in the disconnected state the WiMAX network interface consumes around 3.5 times more energy. This behavior is mainly related with the USB CPE internal design, since it should be possible to save more energy when disconnected, as shown when using the WiFi USB CPE.

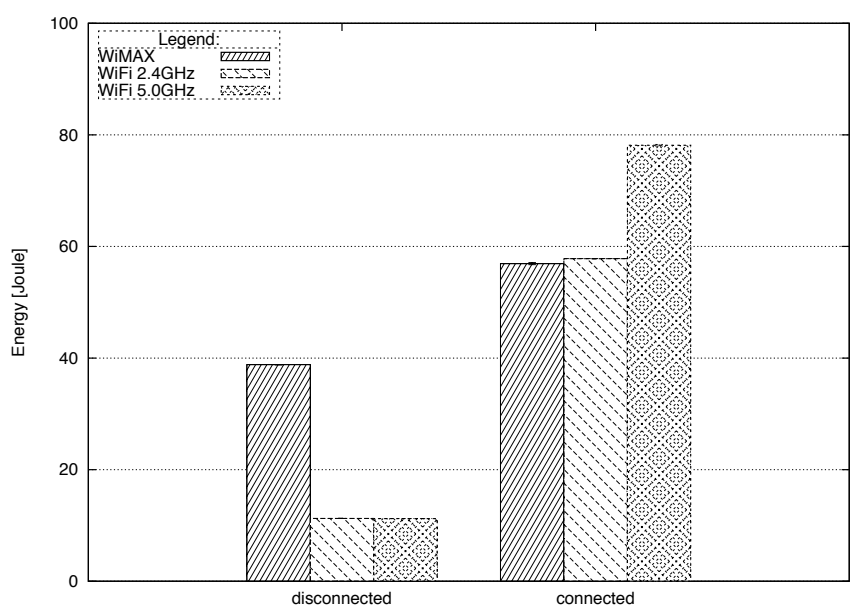

Fig. 5. Energy consumption in disconnected and connected states

The WiMAX is not the most energy demanding technology when connected to the network, which is a point to take into consideration, given the distance between the "Mobile
Node" and the BS, which is higher than the distance in the WiFi testbed. There is a clear tradeoff between the distance supported by the WiMAX technology and the energy consumption, as it is also able to communicated using long distance. The energy saved in the disconnected state compared with the correspondent connected state is $31.83 \%, 80.91 \%$ and $85.65 \%$, respectively for WiMAX, WiFi $2.4 \mathrm{GHz}$ and $\mathrm{WiFi}$ $5.0 \mathrm{GHz}$

The time needed to switch on or off the network interface is important, as together with the energy consumption in the previously analyzed states, it can be used on future energy optimization strategies. Figure 6 shows the time required to switch-on and switch-off the network interfaces. The time

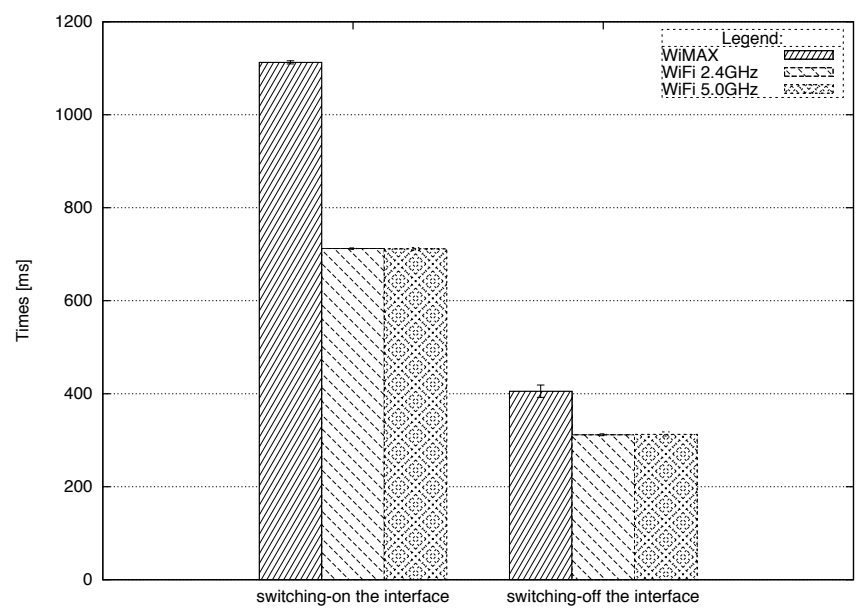

Fig. 6. Time needed to switching-on/off the USB network interfaces

needed to switch-on both WiFi interfaces is around $712 \mathrm{~ms}$, while WiMAX interface takes $1112 \mathrm{~ms}$ to connect to the network. The switching-off procedure of the WiMAX interface takes around $400 \mathrm{~ms}$, whereas both WiFi interfaces use less $100 \mathrm{~ms}$.

Figures 7, 8, 9 show the transitions between the already defined states, respectively for $\mathrm{WiFi} 2.4 \mathrm{GHz}$, WiFi $5.0 \mathrm{GHz}$ and WiMAX. This experiment was done using the following action sequence:

1) State $=$ Disconnected

2) Action: wait for 3 seconds

3) Action: Connect

4) State $=$ Connected

5) Action: wait for 3 seconds

6) Action: DHCP request

7) Action: wait for 10 seconds

8) Action: Disconnect

9) State $=$ Disconnected

Since the total running time is lower than 43 seconds, the $50 \mathrm{~K}$ samples rate was used. In this study, the employment of higher precision is necessary to represent all the small power fluctuations of the system. However, due the very small power fluctuations captured with this higher rate, the usage of a smooth technique to depicted the values is required. 
Therefore, the results presented in the following figures are using a moving average with a window of 1000 samples.

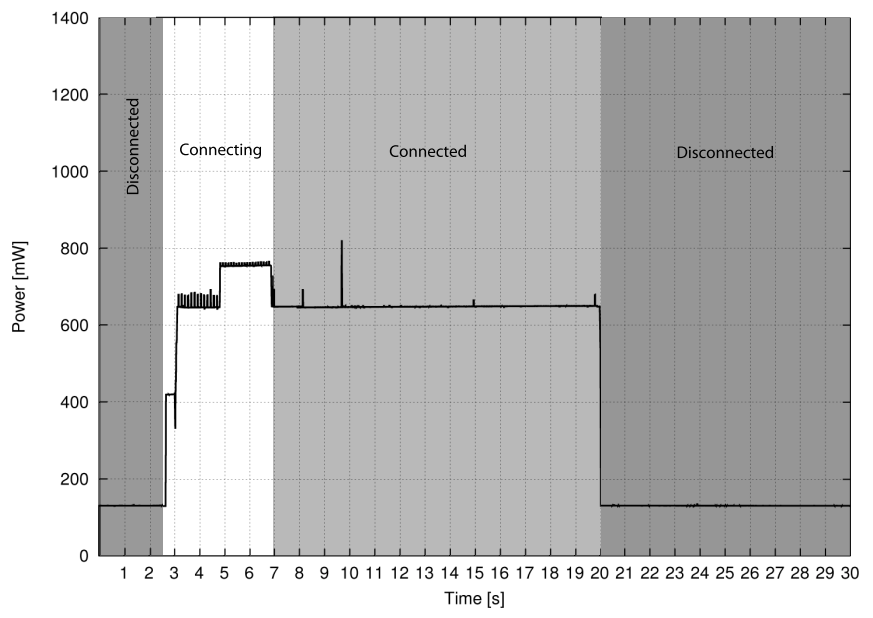

Fig. 7. WiFi $2.4 \mathrm{GHz}$ - states transition

Both WiFi $2.4 \mathrm{GHz}$ and WiFi $5.0 \mathrm{GHz}$ have the same behavior, and all the state transitions are clearly visible. The Dynamic Host Configuration Protocol (DHCP) request 3 seconds after being connected (i.e, at Time $=10 \mathrm{~s}$ ) is also observable in both scenarios.

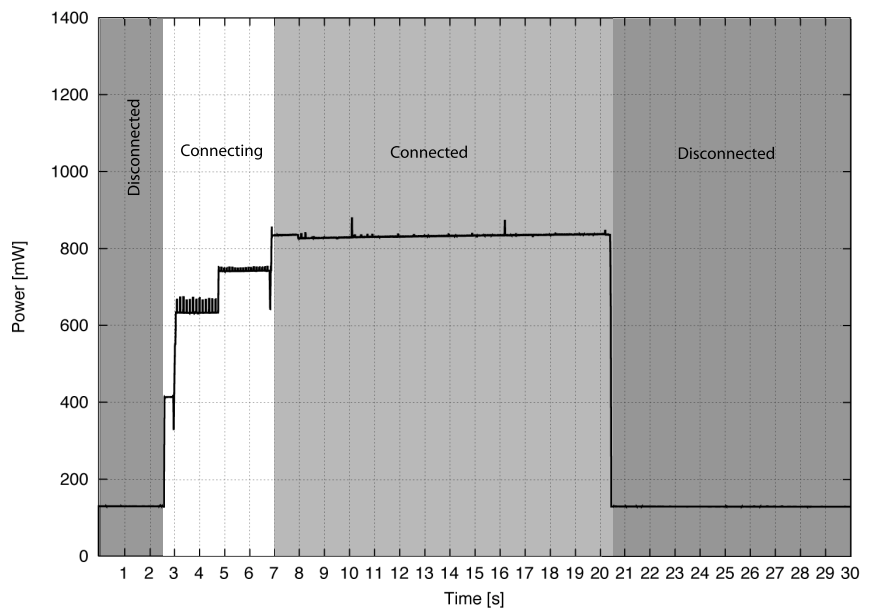

Fig. 8. WiFi $5.0 \mathrm{GHz}$ - states transition

As depicted in Figure 9, the state transitions when using WiMAX are not so smooth, but still well perceptible. The used WiMAX CPE is more power demanding when changing from the disconnected to the connected state. Additionally, there are more power variations during the connected state, when compared with WiFi scenarios. The WiMAX CPE takes longer time to change from the connected to the disconnected state, which can be a drawback when employing energy optimization techniques.

\section{Assessment of packet size impact}

This subsection shows the impact of the packet size in the energy consumption in the studied technologies. The tests

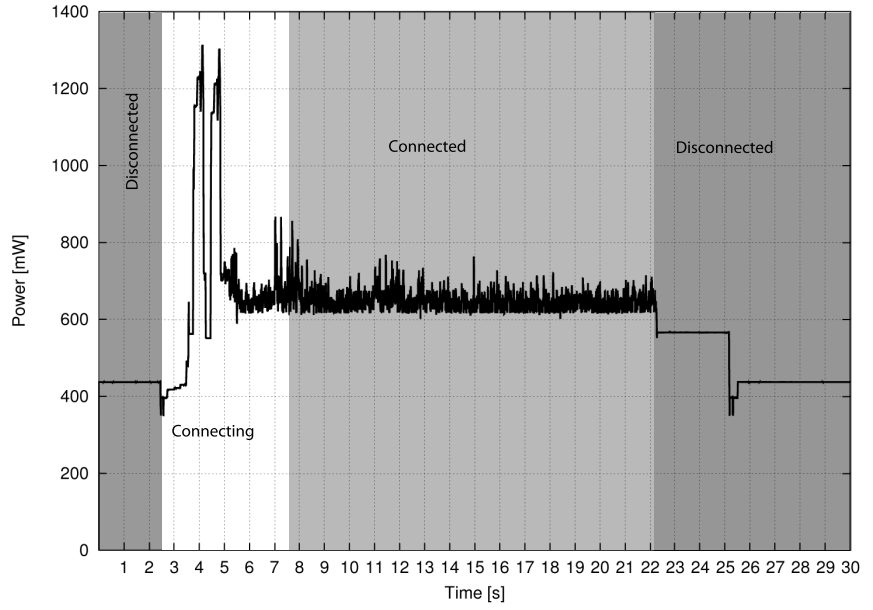

Fig. 9. WiMAX - states transition

were performed using a Constant Bit Rate (CBR) flow using a fixed interval of 100 packets. The energy values depicted in the following figures are calculated taking into account only relevant 90 seconds of the experiments, as already explained in the beginning of the section.

Figure 10 shows the total energy consumed by the USB CPEs to transmit or receive all the 9000 packets (i.e. 100 packets per second during 90 seconds). As expected the energy needed to transmitting a packet is higher than to receive for all studied technologies in almost all the cases. There are some fluctuations in WiMAX, which can be explained by the need of performing antenna transmission power adaptations, due to the distance from the Base Station. By analyzing the error bars in the WiMAX line, it is possible to notice the uncertainty.

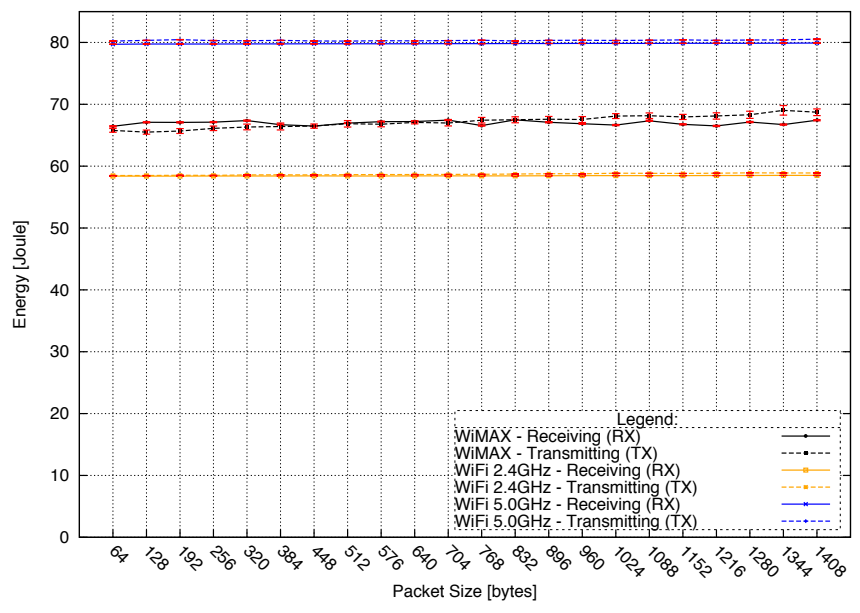

Fig. 10. Total energy consumed with different packet sizes

The WiFi $5.0 \mathrm{GHz}$ is the most energy demanding technology for both transmitting and receiving, which matches the behavior in the connected state showed previously. The WiFi $2.4 \mathrm{GHz}$ uses approximately 58 Joule to transmit 9000 packets of 1024 bytes, while the WiFi $5.0 \mathrm{GHz}$ needs to employ 22 
Joule to perform the same task, and in both the uncertainly with the $95 \%$ confidence interval is only around 0.02 Joule. In the same scenario the WiMAX technology spends about 68 Joule (uncertainly is 0.04 Joule). The energy consumed by the WiMAX and the WiFi $2.4 \mathrm{GHz}$ when connected only (i.e. no data is being transferred) (see Figure 5) is roughly the same, but when transmitting or receiving data, WiMAX needs considerable more energy. Therefore, it is important to analyze the direct impact of the packet size in the energy behavior. As a result, the energy consumed in the connected state were subtracted from the total total energy consumed when transmitting (or receiving) data with the different packet sizes. By performing this operation, the resulting data, depicted in Figure 11, show the overhead on the energy consumption caused only by the data transferred. This approach is feasible, as all the experiments were done in the same conditions and had exactly the same duration and repetitions. Additionally, there is no impact of possible sleep or idle periods, as these modes were deactivated during in the experiments.

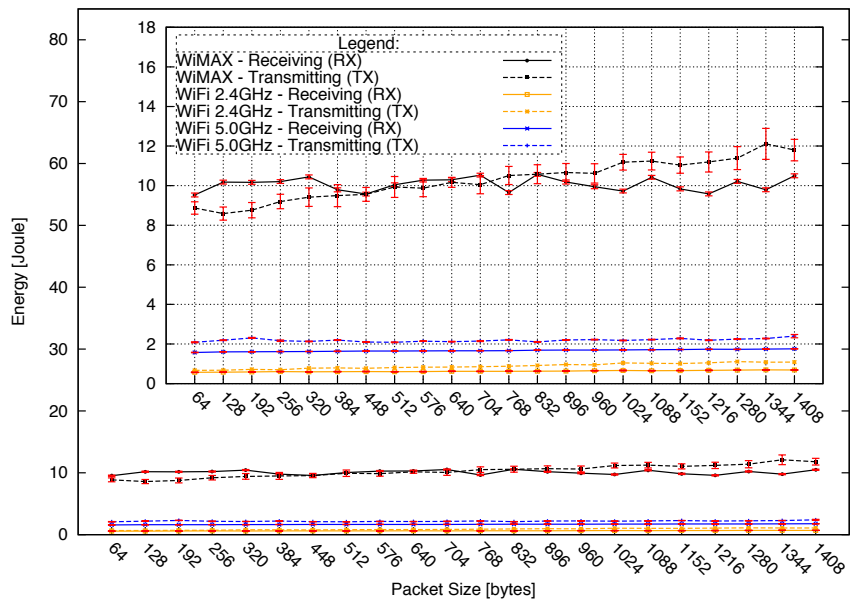

Fig. 11. Energy consumption overhead caused by different packet sizes

As depicted in Figure 11, when considering only the overhead of transmitting data, the WiMAX is the most energy demanding technology. The energy consumption overhead caused in WiMAX for receiving packets of 1408 bytes is around 10.50 Joule, while in WiFi $2.4 \mathrm{GHz}$ and WiFi $5.0 \mathrm{GHz}$ the overhead is, respectively, 0.69 and 1.76 Joule. Nonetheless, it is important to highlight the capabilities of WiMAX technology to transfer data over longer distances.

\section{Assessment of the packet rate impact}

This subsection describes the impact of the packet rate on the energy consumption of each studied technology. The assessment of the packet rate was done using a fixed packet size of 1024bytes, and varying the packet rate from 50 to 1000 packets per second. Again, all the experiments represent the energy consumed during the relevant time in each experiment, and use the same confidence interval and number of runs as in the previous sections.

Figure 12 shows the relationship between the total energy consumed and the packet rate. The WiMAX technology was only assessed until the 500 packets interval, due to the maximum thoughtput support in the link. The energy consumed

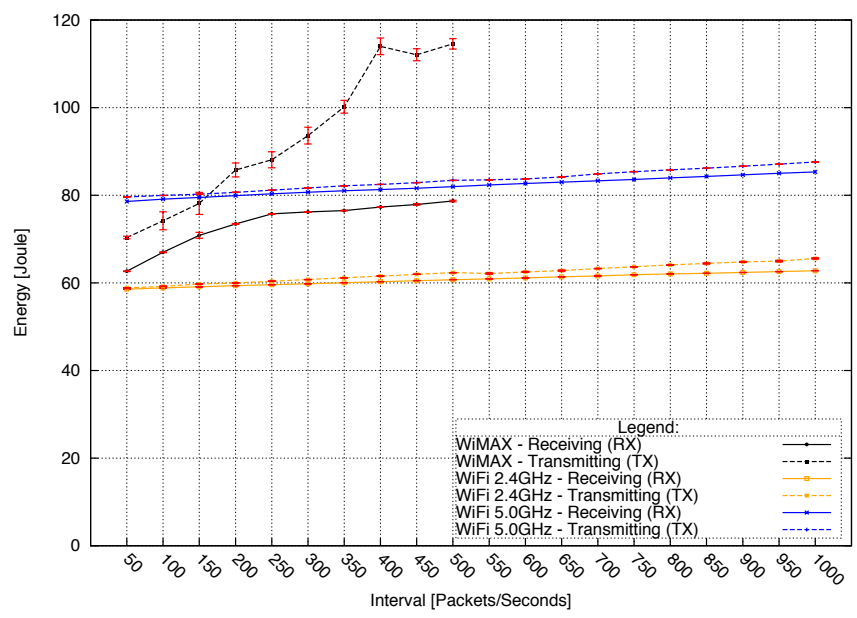

Fig. 12. Energy consumed using different packet rates

for transmission is always higher than for reception for all technologies. In both WiFi scenarios the energy difference between transmission and reception is constant and there is no significant difference between the two states. In contrast the WiMAX scenario shows considerable difference between the energy required to transmit and to receive, especially when using higher packet rates (e.g., 500 packets). By analyzing these results, one can conclude that WiMAX suffers more from the impact of an increased packet rate than both WiFi technologies.

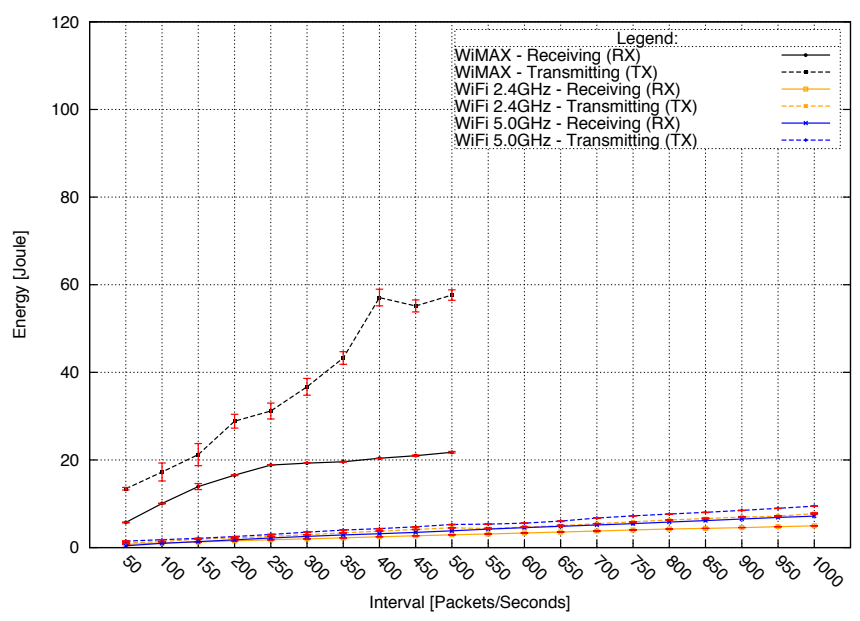

Fig. 13. Energy consumption overhead caused by different packet rates

In Figure 13, the same information is plotted, but the energy consumed in the connected state during the experiment duration was removed, as the Section III-C. The increase of of energy consumption in the WiMAX is nearly exponential, while it is linear in both WiFi scenarios. 


\section{RELATED WORK}

The research question regarding the energy-efficient communication is strongly related to the hardware energy consumption itself, which has a significant impact in the overall results and various studies in the literature addressed the problem by measuring total energy consumption of the enduser device. Although these techniques can be a feasible approach to analyze these systems when compared with the challenge to perform accurate theoretical models for simulation, they do not measure accurately the energy consumed only by the network interface. Balasubramanian et al. [11] have studied the energy consumption in mobile phones with multiple network interfaces, where the main goal was to evaluated the energy-efficient of 3G, GSM and WiFi. Their main contribution is the development of a protocol that reduces the energy consumption of the applications by scheduling the transmission, named TailEnder. Wang and Manner [7] used an Android based phone, and tested the energy consumption using Enhanced Data rates for GSM Evolution (EDGE), High Speed Packet Access (HSPA) and WiFi wireless technologies. The impact of packet size and packet rate were addressed in the study, but only the total energy consumed by the device was measured, which is a clear drawback when trying to optimize the network protocols or applications. Additionally, the study was done using only a specific phone model, which does not exclude the possibility of direct impact of the phone board implementation of the measured energy values.

Rice and Hay [8] proposed a methodology to measure the energy consumption of mobile phones IEEE 802.11 (WiFi) interface, by replacing the battery with a personalized plastic battery holder, which allows an accurate measurement within the phone real energy circuit. To avoid the rapid energy consumption changes caused by the high-frequency components of the mobile phones, the measurement system employs also a high-precision resistor. The study encompasses batch test operations with different mobile phones. The authors argues that the mobile phone itself has some influence in the energy consumption. Their results highlight the work to be done concerning the energy consumption optimization on the mobile devices, by improving the DHCP behavior and proof the contribution of this enhancement to device energy saving. Additionally, one of their main remarks argue that the best energy aware approach to transmit data efficiently over IEEE 802.11 are directly affected by the mobile phone model and operating system. While this work is able to measure accurately the mobile phone energy consumption behavior, it is not able to perform an accurate evaluation of the IEEE 802.11 impact in mobile phone, since the various mobile phone tested seems to have different behaviors, namely when employing different operating systems or hardware in phones.

Shih et al. [12] have developed a technique to increase the battery time when using VoIP calls (only for this application) that is able to shutdown the wireless card/radio when it is not in use. Although the employed technique depends on the application, it shows the potential of analyzing the network interfaces available states to perform application adaptation. To the best of our knowledge, this paper is proposes an original methodology to assess the energy consumption of cloud ready devices, which can be employed in all USB network interfaces and consequently able to measure the energy consumed by the MAC and PHY layers. Moreover, although the Mobile WiMAX energy consumption was already studied in the literature in some theoretical works [13] [14], there is a lack of testbed experimental evaluation concerning the new broadband wireless access networks, which will play a crucial role in the development of new cloud computing services.

\section{CONClusion}

Energy efficiency in end-user devices is a key factor for the acceptance of new cloud computing services, as the devices have to run for a long time. Nevertheless, energy is also an important aspect in the quality perceived by the endusers, since the tradeoff between employed energy saving mechanisms and perceived quality, especially in multimedia based applications, has to be taken into account. All these considerations become even more important in the presence of heterogeneous network ready devices, together with stronger needs regarding mobility, which raise also more difficulties when performing energy optimization.

This paper has proposed an empirical methodology to assess energy consumption of a network interface, using high precision measurement hardware. By using the developed methodology in three different cloud computing access scenarios, it was possible to depict important relationships between the application network related design and the energy spent, namely by analyzing the impact of packet size and packet rate. Moreover, the energy impact of the network technologies states were investigated, and the results showed the importance of an accurate manipulation of those states in order to enhance energy efficiency of the entire system.

Concerning the studied technologies, the results presented the worst energy efficiency for WiFi $5.0 \mathrm{GHz}$ when compared with WiFi $2.4 \mathrm{GHz}$ and WiMAX. Nevertheless, considering only the overhead caused by the network transmissions, WiMAX is the most energy demanding technology in almost all scenarios. The higher energy cost of upcoming $4 \mathrm{G}$ network technologies should be considered, since the tradeoff between throughput and energy may not be the best for long usage, as shown by the results of this study.

In future work, the empirical data obtained, together with additional experiments to be performed, can be used for the development of software-based energy models, which would optimize the energy consumption, while improving the enduser perceive QoE.

\section{ACKNOWLEDGMENT}

This work was partially supported by the Portuguese National Foundation for Science and Technology (FCT) through a Doctoral Grant (SFRH/BD/66181/2009) and by a Short-Term Scientific Mission from the COST Action IC0906 Wireless Networking for Moving Objects (WiNeMO). 
Thanks also to EDPWiMAX project, funded by EDP Distribuição S.A., and to Paulo Simões.

\section{REFERENCES}

[1] C.-S. Lee and D. Knight, "Realization of the next-generation network," Communications Magazine, IEEE, vol. 43, no. 10, oct. 2005.

[2] L. M. Vaquero, L. Rodero-Merino, J. Caceres, and M. Lindner, "A break in the clouds: towards a cloud definition," SIGCOMM Comput. Commun. Rev., vol. 39, pp. 50-55, December 2008. [Online]. Available: http://doi.acm.org/10.1145/1496091.1496100

[3] I. Akyildiz, J. Xie, and S. Mohanty, "A survey of mobility management in next-generation all-ip-based wireless systems," Wireless Communications, IEEE, vol. 11, no. 4, pp. 16 - 28, aug. 2004.

[4] S. Mohanty and I. F. Akyildiz, "A cross-layer (layer $2+3$ ) handoff management protocol for next-generation wireless systems," IEEE Transactions on Mobile Computing, vol. 5, pp. 1347-1360, October 2006. [Online]. Available: http://dx.doi.org/10.1109/TMC.2006.142

[5] Q. He, "A fuzzy logic based vertical handoff decision algorithm between wwan and wlan," in Networking and Digital Society (ICNDS), 2010 2nd International Conference on, vol. 2, may 2010, pp. $561-564$.

[6] N. Nasser, S. Guizani, and E. Al-Masri, "Middleware vertical handoff manager: A neural network-based solution," in Communications, 2007. ICC '07. IEEE International Conference on, june 2007, pp. 5671 -5676.

[7] L. Wang and J. Manner, "Energy consumption analysis of wlan, $2 \mathrm{~g}$ and $3 \mathrm{~g}$ interfaces," in Green Computing and Communications (GreenCom), 2010 IEEE/ACM Int'l Conference on Int'l Conference on Cyber, Physical and Social Computing (CPSCom), dec. 2010, pp. $300-307$.
[8] A. Rice and S. Hay, "Measuring mobile phone energy consumption for 802.11 wireless networking," Pervasive Mob. Comput., vol. 6, pp. 593-606, December 2010. [Online]. Available: http://dx.doi.org/10.1016/j.pmcj.2010.07.005

[9] "Standard digital interface for programmable instrumentation - part 2: Codes, formats, protocols and common commands (adoption of (ieee std 488.2-1992)," IEC 60488-2 First edition 2004-05; IEEE 488.2, pp. $1-261,2004$.

[10] K. Etemad, "Overview of mobile wimax technology and evolution," Communications Magazine, IEEE, vol. 46, no. 10, pp. 31 -40, oct 2008.

[11] N. Balasubramanian, A. Balasubramanian, and A. Venkataramani, "Energy consumption in mobile phones: a measurement study and implications for network applications," in Proceedings of the 9th ACM SIGCOMM conference on Internet measurement conference, ser. IMC '09. New York, NY, USA: ACM, 2009, pp. 280-293. [Online]. Available: http://doi.acm.org/10.1145/1644893.1644927

[12] E. Shih, P. Bahl, and M. J. Sinclair, "Wake on wireless: an event driven energy saving strategy for battery operated devices," in Proceedings of the 8th annual international conference on Mobile computing and networking, ser. MobiCom '02. New York, NY, USA: ACM, 2002, pp. 160-171. [Online]. Available: http://doi.acm.org/10.1145/570645.570666

[13] J. Baliga, R. Ayre, K. Hinton, and R. Tucker, "Energy consumption in wired and wireless access networks," Communications Magazine, IEEE, vol. 49, no. 6, pp. $70-77$, june 2011.

[14] Y. Zhang, Y. Xiao, and V. Leung, "Energy management analysis and enhancement in ieee 802.16e wirelessman," Vehicular Technology, IEEE Transactions on, vol. 58, no. 7, pp. 3738 -3752, sept. 2009. 